\title{
Operations of a non-stellar object tracker in space
}

Riis, Troels; Jørgensen, John Leif; Betto, Maurizio

Published in:

Proceedings of the IEEE Aerospace Conference

Link to article, DOI:

10.1109/AERO.1999.793150

Publication date:

1999

Document Version

Publisher's PDF, also known as Version of record

Link back to DTU Orbit

Citation (APA):

Riis, T., Jørgensen, J. L., \& Betto, M. (1999). Operations of a non-stellar object tracker in space. In Proceedings of the IEEE Aerospace Conference (Vol. 2, pp. 105-111). IEEE. https://doi.org/10.1109/AERO.1999.793150

\section{General rights}

Copyright and moral rights for the publications made accessible in the public portal are retained by the authors and/or other copyright owners and it is a condition of accessing publications that users recognise and abide by the legal requirements associated with these rights.

- Users may download and print one copy of any publication from the public portal for the purpose of private study or research.

- You may not further distribute the material or use it for any profit-making activity or commercial gain

- You may freely distribute the URL identifying the publication in the public portal

If you believe that this document breaches copyright please contact us providing details, and we will remove access to the work immediately and investigate your claim. 


\section{Operations of a Non-stellar Object Tracker in Space}

\author{
Troels Riis \\ Technical University of Denmark \\ Department of Automation \\ Bld. 327 \\ 2800 Lyngby \\ Denmark \\ +45253439 \\ tr@iau.dtu.dk
}

\author{
John L. Jørgensen \\ Technical University of Denmark \\ Department of Automation \\ Bld. 327 \\ 2800 Lyngby \\ Denmark \\ $+45253448$ \\ jlj@iau.dtu.dk
}

\author{
Maurizio Betto \\ Technical University of Denmark \\ Department of Automation \\ Bld. 327 \\ 2800 Lyngby \\ Denmark \\ +45253439 \\ mbe@iau.dru.dk
}

Abstract - The ability to detect and track non-stellar objects by utilizing a star tracker may seem rather straight forward, as any bright object, not recognized as a star by the system is a non stellar object. However, several pitfalls and errors exist, if a reliable and robust detection is required.

To test the operation, performance and robustness of such a function, the Autonomous Vision System (AVS), a fully autonomous star tracker that has flown onboard the Teamsat was equipped with several advanced features. These features included a non-stellar object detection and tracking module and an image and science data compression module.

This paper describes the AVS, sensitivity, and possible detection ranges for various objects. The general description is followed by examples of images and tracking series obtained by the AVS on Teamsat.

\section{TABLE OF CONTENTS}

\section{TEAMSAT OVERVIEW}

2. AVS AND DETECTION SENSITIVITY

3. TRACKING RANGE OF GEO-SATELLITES

4. ACHIEVED ORBIT

5. TRACKED OBJECTS

6. CONCLUSION

7. REFERENCES

8. BIOGRAPHY

\section{TEAMSAT OVERVIEW}

The second Ariane 5 prototype vehicle to be launched by ArianeSpace was designed to lift two test payloads Maqsat$B$ and Maqsat-H into Geostationary Transfer Orbit (GTO). ESTEC, a technical facility of the European Space Agency (ESA), applied for, and got approved that part of the mass of the Maqsat-H could be used for a technological demonstration sub-payload. The objectives of ESTEC were twofold. Firstly, to get practical experience with a BetterFaster-Cheaper type mission. And secondly, to provide space departments of European universities and companies with an opportunity to get advanced technologies and equipment tested in space.
The result of this effort was Teamsat. This sub-payload, weighing $350 \mathrm{~kg}$, was to be developed, assembled, tested and operationally verified in precisely 1 year, all at marginal cost.

Teamsat was to be launched into a geostationary transfer orbit by the Ariane 5 vehicle. At or near apogee several geostationary satellites were expected to come within the detection range of the AVS, whence the detection and tracking of these was a major objective for the flight. Furthermore, Teamsat featured a small sub-satellite YES (Young Engineers Satellite) that was to be deployed late in the mission. Another objective was therefore to track YES during and after deployment for as long as possible.

The non-nominal operation of the first stage of the launch vehicle resulted in an apogee too low to enable the detection of geostationary satellites. However, as the launcher used all fuel to achieve the highest possible apogee, no fuel was left for attitude maneuvers during deployment let alone for the depletion burn. Hence several objects were moving around the vicinity of Teamsat throughout and provided for an excellent set of test objects for the AVS.

\section{AVS AND DETECTION SENSITIVITY}

The AVS differ mainly from other star trackers by being fully autonomous. Based on a powerful microprocessor, the AVS is a perfect platform for doing complex image analysis. It consists of a small camera head unit (CHU) and a data processing unit (DPU). Depending on the shielding thickness, the masses of the CHU and DPU are only 180 and 700 grams respectively.

The separation of the CHU and DPU is primarily due to three reasons. 1) Minimal disturbance, since the $\mathrm{CHU}$ can be placed far from the DPU. 2) Possibility of direct integration of the CHU with a science instrument in an optical bench for optimal attitude precision. 3) Flexibility in satellite bus integration due to the small size.

The star tracker function of the AVS is based on two different attitude determination algorithms. 
The first is the so-called "Initial Attitude Acquisition" algorithm which solves the "Lost in Space" situation. It uses triplets of stars to perform a very fast search for matches in a special compiled star database. The output of this algorithm is the attitude with a precision of approximately $1 / 100$ of a degree.

The second algorithm is called "A-priori Attitude Determination". It uses the previously determined attitude to determine the current attitude. This routine is always invoked first. If it fails, which will be the case if it is the first image since boot, or if the attitude drift from the previous image is too large, then the "Initial Attitude Acquisition" algorithm will be invoked followed by the "Apriori Attitude Determination" algorithm again.

Both algorithms are very fast, in the range of $50-100 \mathrm{~ms}$ depending on which portion of the sky is imaged. This makes it possible always to perform any sequence of the two algorithms if the AVS is running at a rate of $1-4 \mathrm{~Hz}$. If the attitude drift from the previous image is less than approximately 1 degree, which normally is the case, then only the "A-priori Attitude Determination" will be performed. Therefore it is possible to operate the AVS at higher frequencies.

The star catalogue is generated from the Hipparcos Catalogue using the proper motion for the epoch of the mission.

The AVS uses only the lowest 8 bit of 14 bit dynamic range of the CCD. If more light reaches a pixel than corresponds to the 8 bit $-P_{255}$ (Def.: $D N=$ Digital Number [0:255]), the pixel value will remain $P_{255}$. This means it takes an illumination resulting in a value of $64 \cdot P_{25 s}$ for a pixel to saturate and spill over into the CCDs anti-blooming channels. These can handle another factor of 100 before the charge starts to spill over to neighboring pixels. To avoid blooming disturbances on neighboring pixels, the illumination of a single pixel must be kept below approximately $5000 \cdot \mathrm{P}_{255}$. Sirius at $\mathrm{m}_{\mathrm{v}}=-1.4$ is only at $150 \cdot P_{255}$, while Jupiter and Venus at $m_{v}=-4$ is at $1600 \cdot P_{255}$. Blooming is only a problem in the sense that it takes longer time to process the image and the attitude precision is getting reduced.

An example of this is shown in Figure 1. It shows an image of a $3 / 4$ full moon in the field of view. The image is taken during a test at the JPL facility Table Mountain Observatory north of Los Angeles, USA. The ASV does take a little longer time to process the image, but it is still quite capable to determine the attitude from the stars in the remainder of the image. The moon itself is only about $1 / 20^{\text {th }}$ the width of the image. The rest of the light around the moon is due to the atmosphere spreading, blooming, and the $2^{\text {nd }}$ order effect of the lens. The attitude is determined to be R.A.= 37.627511, Decl. $=2.902540$, and Rot. $=136.736162$.

The dynamic range of the CCD makes it possible to detect fainter stars than $m_{v}=7$. However, a threshold is used in the centroiding algorithm so that stars fainter than $m_{v}=7$ will not be included in the attitude determination. Including fainter stars in the star catalogue will not improve the precision of the attitude. On the contrary, it will only increase the number of stars substantially in the star catalogue. Also the star catalog does not include deep space objects such as galaxies and nebula, which will have to be accounted for if fainter stars were used.

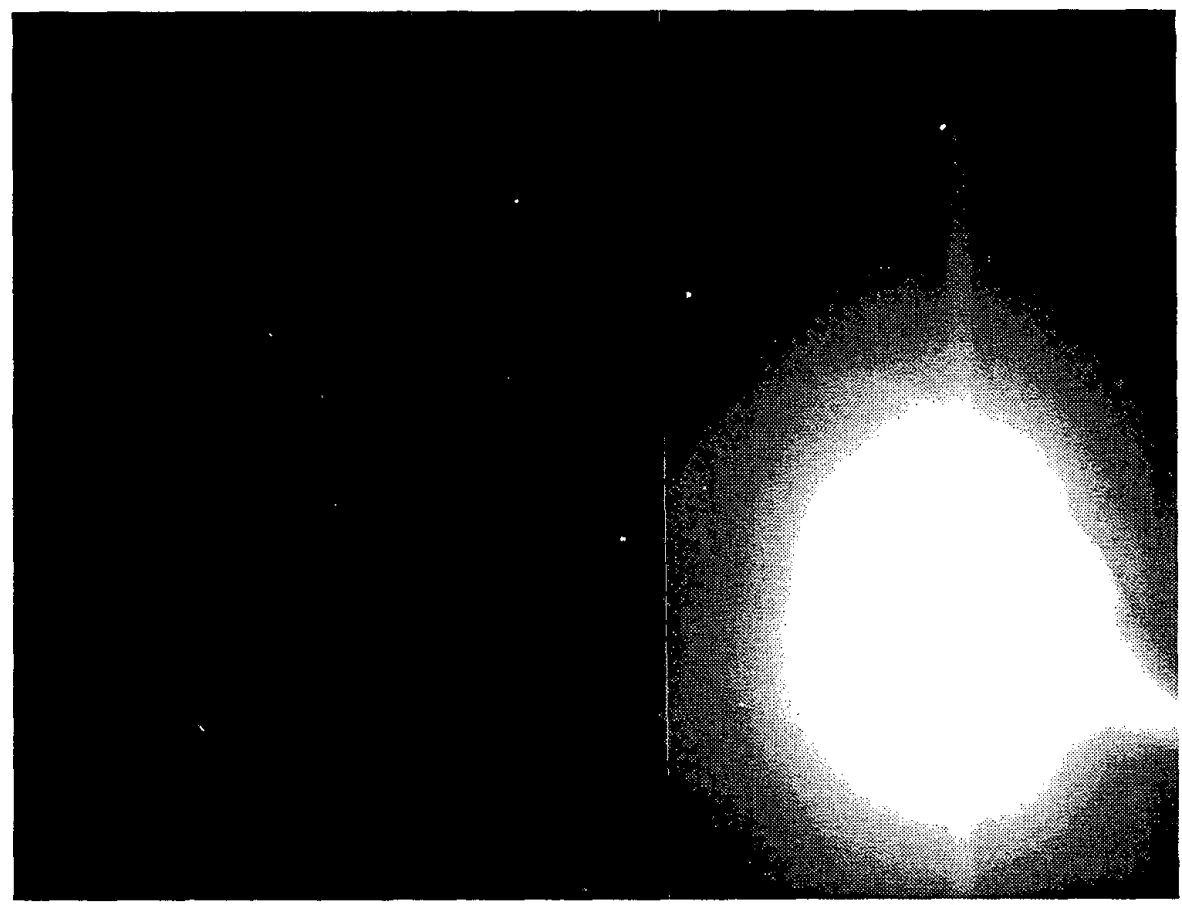

Figure 1. AVS determines the attitude even though the Moon is in the fov. 
Asteroids, comets, planets and satellites will also be detected if they are brighter than $m_{v}=7$. Since Teamsat in GTO would be close to the GEO satellites once every orbit, one of the objectives for AVS was to detect and track these non-stellar objects.

\section{TRACKING RANGE OF GeO-SATELlites}

In order to calculate the tracking range of any non-stellar object in the field of view of the AVS, an estimate of the expected illumination of the object and of the $C C D$ must be made.

The apparent visual magnitude of the sun at the Earth is $\mathrm{m}_{\mathrm{v} \text {,sun }}=-26.8$ and the illumination from the Sun is $\mathrm{E}_{\mathrm{sun}}=$ $1370 \mathrm{~W} / \mathrm{m}^{2}$. A star or an object of magnitude $m_{v}=7$ yields an illumination of:

$$
E_{m_{v}=7}=E_{s u n} \cdot 2.512^{-26.8-7}=41 p W / m^{2}
$$

This means that an object must have an illumination at the CCD of more than $41 \mathrm{pW} / \mathrm{m}^{2}$ in order to be detectable if the AVS threshold is set at $\mathrm{m}_{\mathrm{v}}=7$. The incident solar power $\mathrm{P}_{\text {object }}$ on a object with an area $\mathrm{A}_{\text {object }}$ at the distance $\mathrm{r}_{\text {sun-object }}$ (measured in $\mathrm{AU}$ ) from the sun can be calculated as:

$$
P_{\text {object }}=E_{\text {sun }} \cdot\left(\frac{1 A U}{r_{\text {sun-object }}}\right)^{2} \cdot A_{\text {object }}
$$

The illumination from the object, at the distance $r_{A v s}$, to the AVS lens can be estimated as:

$$
E_{A V S}=\frac{\alpha \cdot v_{f} \cdot P_{o b j e c t}}{2 \pi \cdot r_{A V S}^{2}}
$$

where $\alpha$ is the albedo of the object, and $v_{f}$ is the fraction of the object visible from the AVS. An estimate of the detection range $\mathrm{r}_{\text {object-AVs }}$ of objects from the AVS can then be found as:

$$
r_{\text {object-AVS }}=\sqrt{\frac{\alpha \cdot v_{f} \cdot E_{s u n} \cdot A_{o b j e c t}}{2 \pi \cdot E_{A V S} \cdot r_{s u n-o b j e c t}^{2}}}
$$

For GEO satellites $r_{\text {sun-object }}$ is approximately $1 \mathrm{AU}$ and the satellite have a body area $A_{\text {object }}$ of approximately $15 \mathrm{~m}^{2}$. If the illumination $\mathrm{E}_{\mathrm{AvS}}$ is set equal to the illumination corresponding to a $\mathrm{m}_{\mathrm{v}}=7 \mathrm{star}$, and using an albedo $\alpha=0.7$ and a visible fraction $v_{f}=0.7$, the detection range is approximately $6.300 \mathrm{~km}$. For the ideal case with $\alpha=v_{\mathrm{f}}$ $=1.0$, the detection range is $8.900 \mathrm{~km}$. Table 1 summarizes detection ranges for different values of the combined factor $\alpha \cdot v_{f}$, and the object area $A_{o b j e c t}$.
It can be seen that the detection range varies from approximately $5-10.000 \mathrm{~km}$ depending on the size of the satellite, its orientation and its surface properties. It was therefore planned to have the AVS detect and track the GEO satellites and any other non-stellar object while Teamsat approached apogee in the orbit.

Table 1. Detection range of GEO satellites.

\begin{tabular}{|c|c|c|}
\hline$\alpha \cdot v_{\mathbf{f}}$ & $A_{\text {object }}$ & $r_{\text {object-AVs }}$ \\
\hline 0.5 & 10 & $5.135 \mathrm{~km}$ \\
\hline 0.7 & 10 & $6.080 \mathrm{~km}$ \\
\hline 1.0 & 10 & $7.265 \mathrm{~km}$ \\
\hline 0.5 & 15 & $6.290 \mathrm{~km}$ \\
\hline 0.7 & 15 & $7.445 \mathrm{~km}$ \\
\hline 1.0 & 15 & $8.900 \mathrm{~km}$ \\
\hline 0.5 & 20 & $7.265 \mathrm{~km}$ \\
\hline 0.7 & 20 & $8.595 \mathrm{~km}$ \\
\hline 1.0 & 20 & $10.275 \mathrm{~km}$ \\
\hline
\end{tabular}

\section{ACHIEVED ORBIT}

Due to the anomaly in the first stage of the launcher, the actual orbit achieved by Teamsat was quite different from what was planned. The nominal and achieved orbit parameters are shown in Table 2.

Table 2. Orbit parameters.

\begin{tabular}{|l|c|c|}
\hline & Nominal & Achieved \\
\hline Semimajor axis $(\mathrm{km})$ & 24628 & 19962 \\
\hline Eccentricity & 0.717 & 0.654 \\
\hline Inclination & $7.75^{\circ}$ & $7.77^{\circ}$ \\
\hline R.A. of Sac. Node & $58^{\circ}$ & $58^{\circ}$ \\
\hline Arg. of Perigee & $178^{\circ}$ & $178^{\circ}$ \\
\hline $1^{\text {st } \text { Apogee Altitude }(\mathrm{km})}$ & 35919 & 26639 \\
\hline Perigee Altitude $(\mathrm{km})$ & 580.9 & 528.7 \\
\hline
\end{tabular}

The separation of Teamsat from the launcher also left Teamsat in a very flat spin. The AVS, pointing aft on Teamsat, made the attitude measurements based on onboard star tracking. The measurements were obtained while passing from looking at the earth to the sun. Figure 2 summarizes the Teamsat attitude as determined by the AVS measurements. $\mathbf{L}$ is the angular momentum of Teamsat, $\mathbf{x}$ is the Teamsat symmetry axis, R.A. and DEC are the right ascension and declination to which $\mathbf{L}$ points at the specified time.

Since the apogee of the orbit was about $9.280 \mathrm{~km}$ short of the GEO, it can be seen from Table 1, that only in the case of total reflection from a large satellite would the AVS be able to detect any GEO satellites. Further, the non-nominal spinning of Teamsat caused the AVS to point at the Earth and the Sun for large portions of the time. Due to the design of Teamsat, the AVS was only allowed to be mounted with a very short 5 $\mathrm{cm}$ baffle. Even though the AVS was looking directly in to the Sun for large portions of the time, as soon as it came out of the 


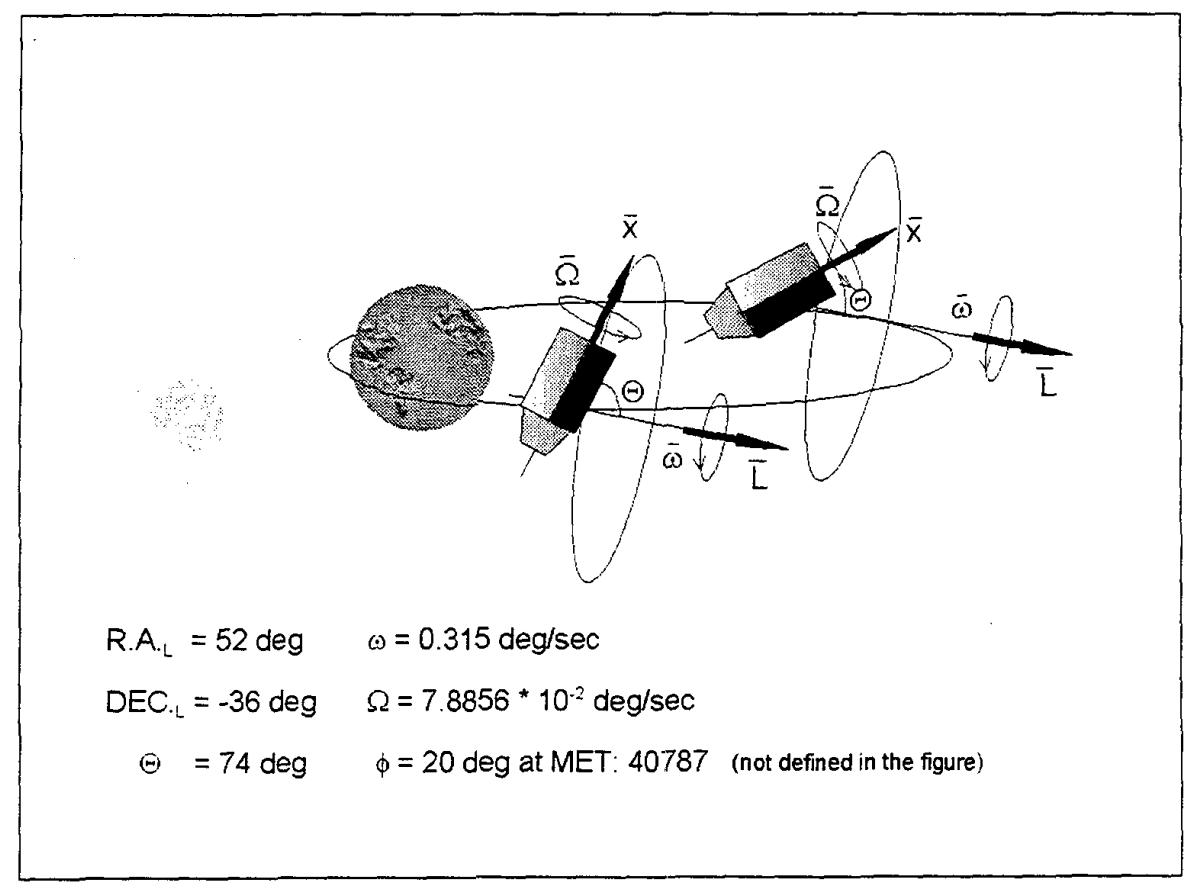

Figure 2. Teamsat attitude as determined by the AVS.

Sun, it just performed an "Initial Attitude Acquisition" and was back on track again. No damage were expected to the CCD by looking at the Sun and none was observed.

\section{TRACKED OBJECTS}

The AVS is equipped with a non-stellar object detector which have been assessed in real-sky tests using earthorbiting satellites as objects. A typical pointing accuracy of 3 " was found with a 25" noise per star at a detection limit of $\mathrm{m}_{\mathrm{v}}=9$. The non-stellar object function may be enabled by command, and the number of objects tracked (up to 200) is user selectable.

As stated earlier, asteroids, comets, planets and satellites will also be detected by the AVS if they are brighter than $m_{v}$ $=7$. The same goes for radiation spots on the CCD. All of these will be detected as outliers since they are not in the star catalogue and therefore rejected during the attitude determination process. Even though the AVS is capable of detecting faint $m_{y}=9$ objects, the compiled star catalogue on board Teamsat only contained stars down to $m_{v}=7$. The AVS do have the capability to inflight upload all software anew including a new star catalogue, so as to handle the increased detection distance to the GEO satellites. Due to the restricted TC bandwidth it was however not possible to do this on Teamsat.

The radiation spots are concentrated on a single or double pixel, and will be located in the same pixel positions in sequences of images, while non-stellar objects will move relative to the stars as well as the pixel position when the

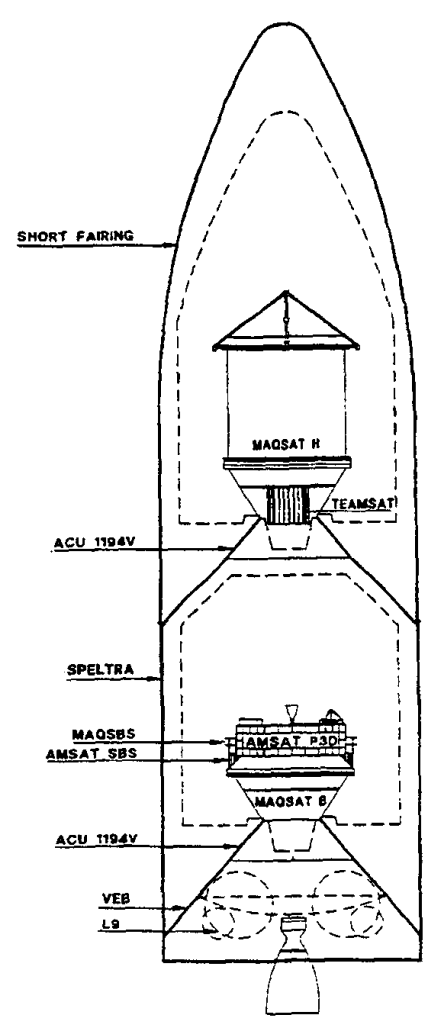

Figure 3. The Ariane 502 launch configuration and the position of Teamsat. 
AVS is spinning. Any object detected, not recognized as "stars" or radiation spots, can then be used as a target to the detection and tracking of non-stellar objects in the field of view of the AVS in sequences of images.

Besides detecting and tracking GEO satellites, the AVS was to track any other object in the vicinity of Teamsat. The launch configuration of the Ariane 502 is shown in Figure 3. The short fairing is ejected at burnout of the first stage and is not expected to be visible for the AVS when it is first turned on. Maqsat- $\mathrm{H}$ was scheduled to seperate from the speltra with the Maqsat-B test satellite inside $48^{\circ}$ after burnout of the second stage (i.e. perigee). The Magsat-H and the speltra was supposed to be oriented in the velocity direction at the time of seperation. The AVS would then have been able to continously track the speltra as it would drift away. Due to the nonnominal launch insertion into the orbit, the satellites were spinning and were oriented almost perpendicular to the velocity vector at the time of seperation. This situation is depicted in Figure 4. The figure also shows the resulting approximate orbits of the seperated objects. The two objects will drift apart for almost half an orbit and then start to approach each other again. Therefore the object should continue to be in the vicinity of Teamsat throughout the mission. Indeed we continued to see the object from time to time depending on the distance and the attitude in the 20 orbits. The expected seperation velocity was $1 \mathrm{~ms}^{-1}$, which would give a maximum seperation of approximately $0.5 \cdot \mathrm{T}_{\text {orbit }} \cdot \mathrm{v}_{\text {seperation }} \cong 15 \mathrm{~km}$.

The first image acquired by the AVS after launch is shown in Figure 5. Part of the light from the Earth can be seen in the upper right corner, and the glare from it is effecting almost half of the image. The stars can be seen as small almost vertical lines due to the high rotation of Teamsat. Radiation spots are seen as small bright dots on a single or double pixel. To the left under the TM dropout can be seen a non-stellar object.

This object is much larger than the stars and the line it draws during the integration time is in a slightly different direction than the stars. The "two parts" of the objects is due to the interlace mode operation of the CCD camera. The object is most probably the speltra with the Maqsat-B. The AVS detected the object and outputted the attitude of the object as seen from the AVS. Assuming the object is the speltra, the distance to it can be estimated to be approximately $1000-1500 \mathrm{~m}$.

Another instrument aboard the Teamsat was the Young Engineers Satellite (YES), which was a small micro-satellite tethered system. It was originally planned to have deployed a $35 \mathrm{~km}$ long tether, after which it should have been ejected from the bottom of Teamsat to re-enter and burn up in the atmosphere. The International Steering Committee for Space Debris gave it a no-go due to risk considerations, which resulted in YES being ejected without the tether. If Teamsat had be launched into nominal attitude in the orbit, YES should have been in the field of view of the AVS, and it should therefore have been detected and tracked by the AVS in a sequence of images.

However, due to the spinning of the Teamsat at ejection time, YES was also not ejected along the orbit direction, causing it too to reappear from time to time in the field of view of the AVS. Figure 6 shows one of the last images taken by the AVS. It shows what is belived to be YES as well as the speltra.

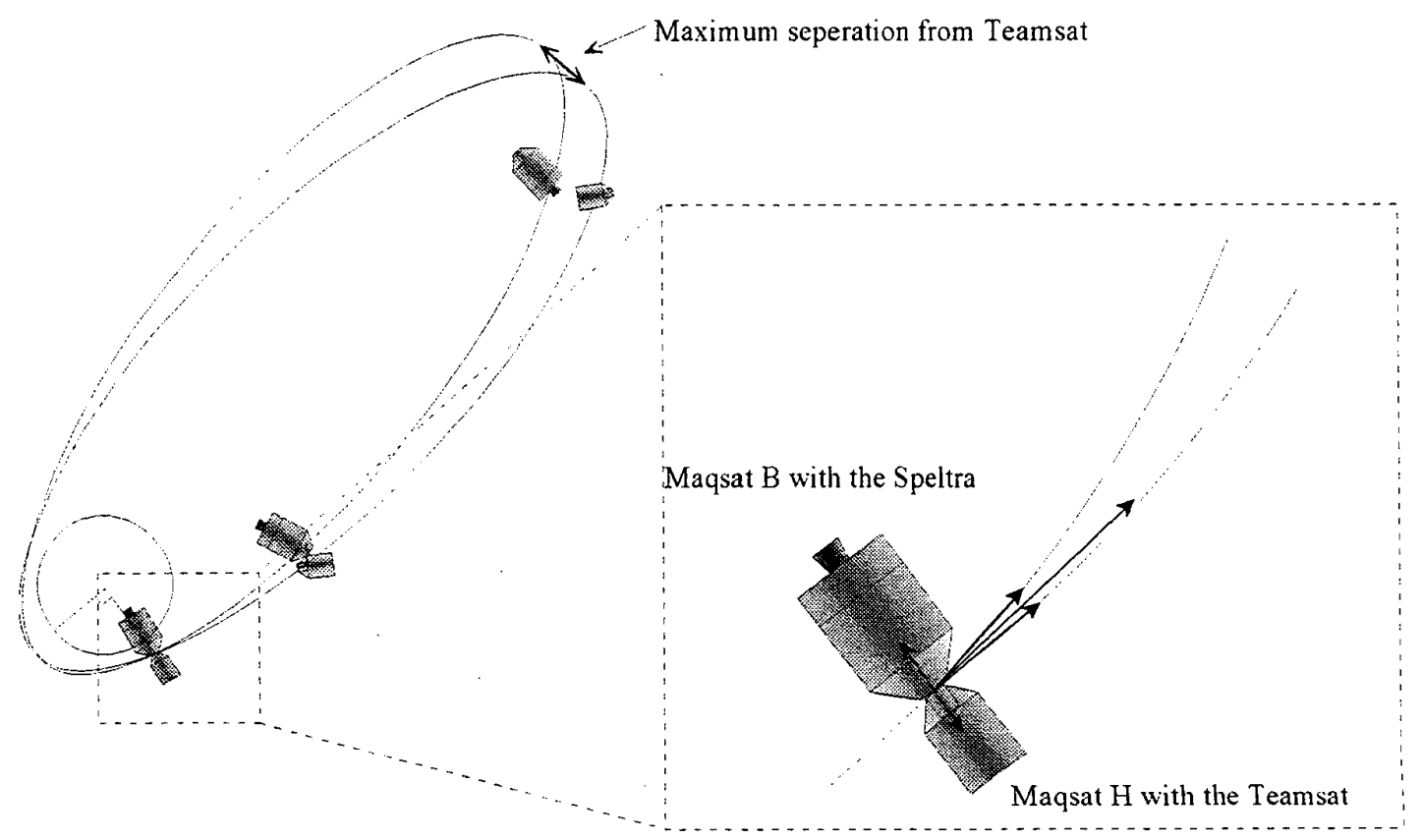

Figure 4. Orbit of Teamsat and Maqsat B after seperation. 


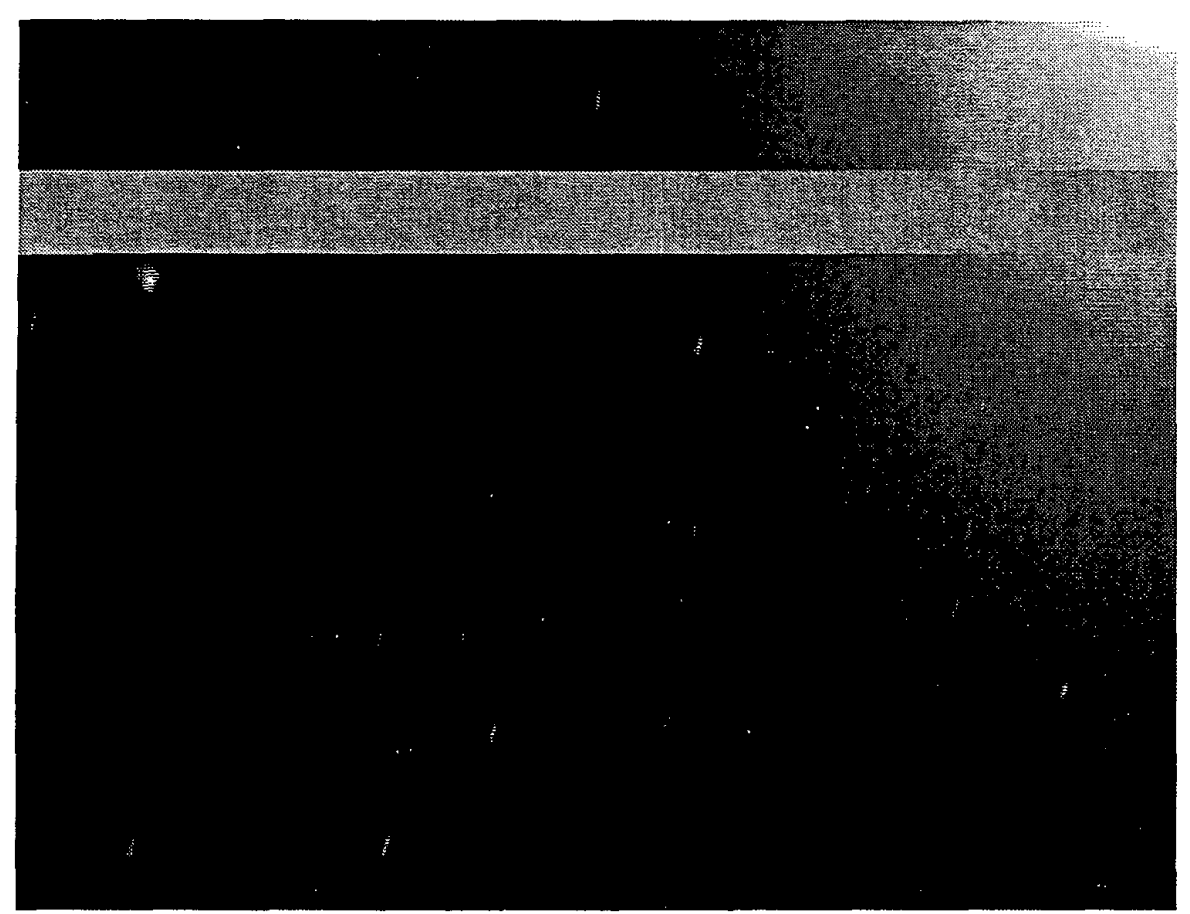

Figure 5. First image acquired by the AVS. The gray band across the image is due to a telemetry dropout between the Teamsat OBDH and the ground EGSE.

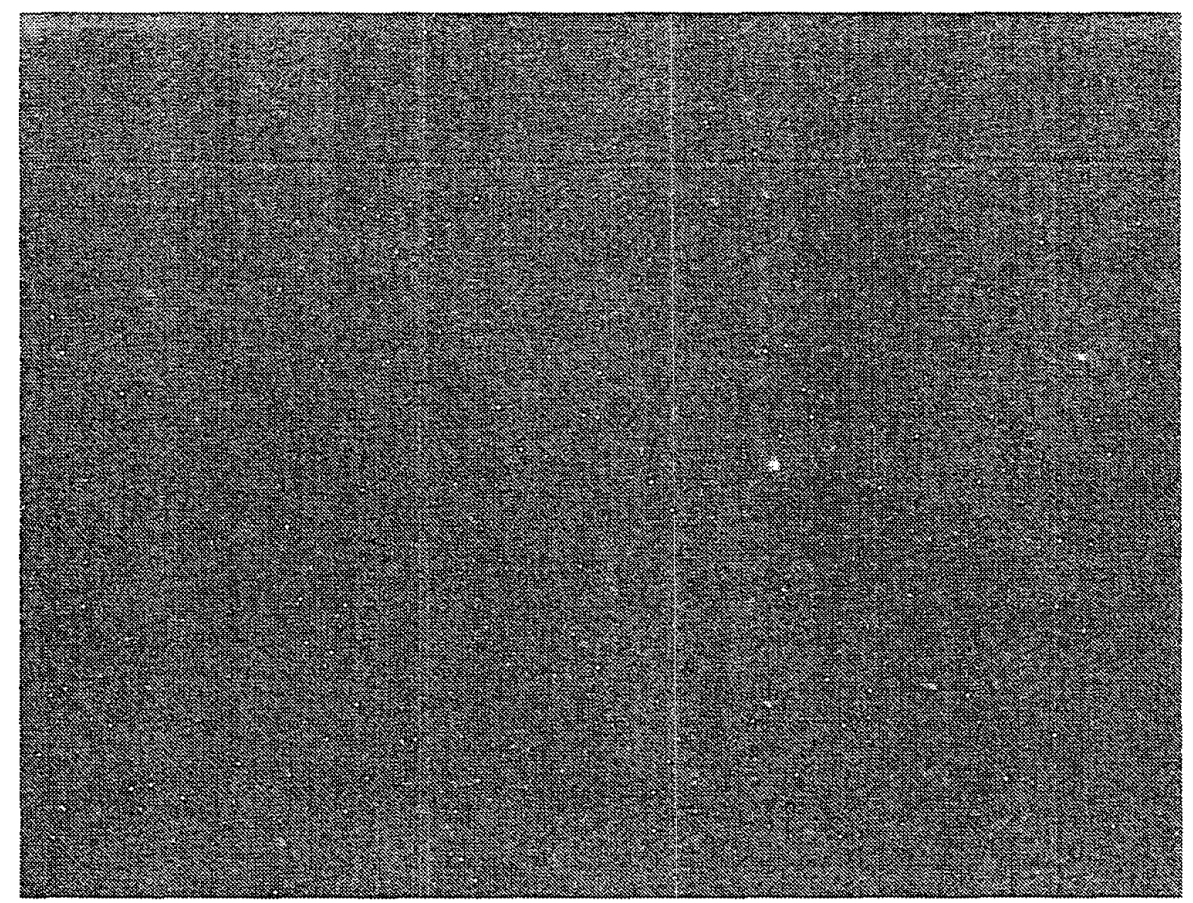

Figure 6. One of the last images acquired by the AVS. 
Note also the increased number of radiation spots in the image in Figure 5 compared to the image in Figure 6. On Teamsat, the thickness of the Camera Head Unit was deliberately made thin, so as to receive a life time radiation dose during the approximately 20 orbits each passing the Van Allen proton belts twice. This was one of the other main goals of the AVS onboard Teamsat so as to get real data for the radiation sensitivity of the $C C D$ chip and camera design.

\section{CONClusion}

The Teamsat was only equipped with $\mathrm{NiCd}$ batteries and no solar cells so the mission duration was limited to approximately 4 days. During this period, the different instruments on the AVS were switched on and off periodically in order to distribute the power equally. This and the fact that the Teamsat satellite was ejected spinning into a non-nominal orbit looking at the Sun for large portions of the time resulted in a non-optimal test sequence for the AVS with respect to accuracy and detection possibilities, but nice testobjects were imaged.

However, the AVS functioned flawlessly. It determined the attitude and attitude dynamics of the Teamsat after the nonnominal orbit insertion. It did not receive any damage, except for the expected radiation effects on the CCD, and kept functioning even though it was pointing at the sun for large portions of the time. It also received the planned life time dose of radiation in order to study the effects hereof.

The AVS was not able to detect the GEO satellites due to the non-nominal orbit, but it reliably detected the objects in the vicinity of Teamsat throughout the mission.

Though the Ariane 502 launch itself was not a perfect mission, for the AVS onboard the Teamsat it proved to operate quite successfully under difficult circumstances.

\section{REFERENCES}

[1] M. Betto, J.L. Jørgensen, T. Riis, "The Autonomous Vision System on Teamsat", IEEE Aerospace Conference, 1999.

[2] J.L. Jørgensen, T. Ruis, M. Betto, "Star tracker and vision system performance in a high radiation environment", IEEE Aerospace Conference, 1999.

[3] John Leif Jørgensen, Allan Read Eisenmann and Carl Christian Liebe, "Autonomous vision in space, on an Advanced Stellar Compass," 1996 SPIE Space Sciencecraft Control and Tracking in the new Millenium, August 6-8 1996. Volume 2810 p. 54-63.

[4] M. Bandecchi, W.J. Ockles, "The Teamsat Experience" ESA bulletin 95, Aug. 1998, p. 132-157

[5] Teamsat Internet homepage at the address: http://www.estec.esa.n//Teamsat

\section{BIOGRAPHY}

Troels Riis is currently a Ph.D. student at the Technical University of Denmark (DTU) in the Space Instrumentation Group (SIG). He has a MSc in Engineering. He is mainly doing research in the areas of nonstellar object detection and tracking, and pattern recognition for the AVS.

John L. Jørgensen is a professor at the department of Automation at the Technical University of Denmark (DTU), where he is head of the Space Instrumentation Group (SIG) that developed the AVS. He has a MSc in Engineering and $a H D$ in business and administration. His main research activities are robust vision systems, star trackers and vision in space

Maurizio Betto is currently a Ph.D student at the Technical University of Denmark (DTU) in the Space Instrumentation Group (SIG) working on the navigation function of the AVS. He has a $M S c$ in Astronomy and worked seven years at the Europen Space Agency in the field of the $g$ idance navigation and control.
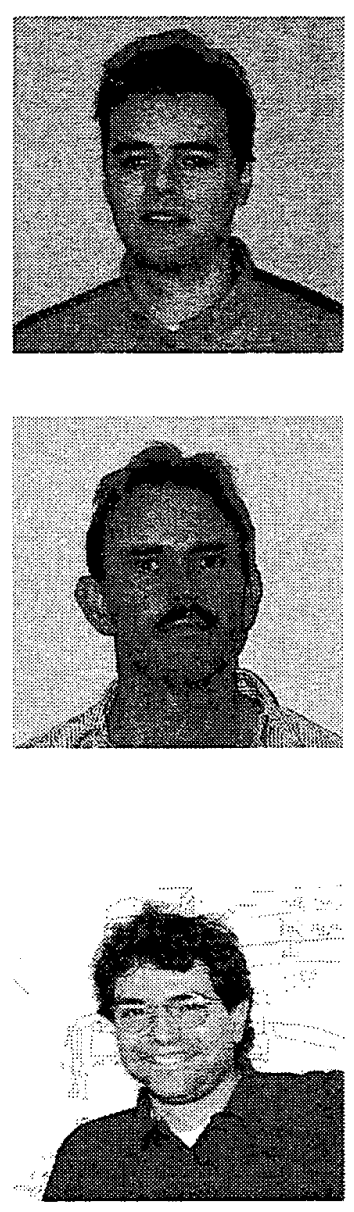